\title{
Community treatment orders for patients with psychosis (OCTET): a randomised controlled trial
}

Tom Burns, Jorun Rugkåsa, Andrew Molodynski, John Dawson, Ksenija Yeeles, Maria Vazquez-Montes, Merryn Voysey, Julia Sinclair, Stefan Priebe

\begin{abstract}
Summary
Background Compulsory supervision outside hospital has been developed internationally for the treatment of mentally ill people following widespread deinstitutionalisation but its efficacy has not yet been proven. Community treatment orders (CTOs) for psychiatric patients became available in England and Wales in 2008. We tested whether CTOs reduce admissions compared with use of Section 17 leave when patients in both groups receive equivalent levels of clinical contact but different lengths of compulsory supervision.
\end{abstract}

Methods OCTET is a non-blinded, parallel-arm randomised controlled trial. We postulated that patients with a diagnosis of psychosis discharged from hospital on CTOs would have a lower rate of readmission over 12 months than those discharged on the pre-existing Section 17 leave of absence. Eligible patients were those involuntarily admitted to hospital with a diagnosis of psychosis, aged 18-65 years, who were deemed suitable for supervised outpatient care by their clinicians. Consenting patients were randomly assigned (1:1 ratio) to be discharged from hospital either on CTO or Section 17 leave. Randomisation used random permuted blocks with lengths of two, four, and six, and stratified for sex, schizophrenic diagnosis, and duration of illness. Research assistants, treating clinicians, and patients were aware of assignment to randomisation group. The primary outcome measure was whether or not the patient was admitted to hospital during the 12-month follow-up period, analysed with a log-binomial regression model adjusted for stratification factors. We did all analyses by intention to treat. This trial is registered, number ISRCTN73110773.

Findings Of 442 patients assessed, 336 patients were randomly assigned to be discharged from hospital either on CTO (167 patients) or Section 17 leave (169 patients). One patient withdrew directly after randomisation and two were ineligible, giving a total sample of 333 patients (166 in the CTO group and 167 in the Section 17 group). At 12 months, despite the fact that the length of initial compulsory outpatient treatment differed significantly between the two groups (median 183 days CTO group vs 8 days Section 17 group, $\mathrm{p}<0.001$ ) the number of patients readmitted did not differ between groups (59 [36\%] of 166 patients in the CTO group vs 60 [36\%] of 167 patients in the Section 17 group; adjusted relative risk $1 \cdot 0[95 \%$ CI $0 \cdot 75-1 \cdot 33])$.

Interpretation In well coordinated mental health services the imposition of compulsory supervision does not reduce the rate of readmission of psychotic patients. We found no support in terms of any reduction in overall hospital admission to justify the significant curtailment of patients' personal liberty.

Funding National Institute of Health Research.

\section{Introduction}

The psychiatric inpatient population has fallen drastically in developed countries since its peak in the mid-1950s. ${ }^{1}$ Patients with psychosis are now routinely treated in the community. Many are subject to repeated compulsory admissions ("the revolving door syndrome") ${ }^{2}$ and the absolute rate of involuntary admissions has increased. ${ }^{3}$ Legislation for compulsory outpatient psychiatric treatment has been widely introduced in the USA, Australasia, some Canadian provinces, the UK, and several other European countries.

Supervised community treatment orders (CTOs) were introduced in England and Wales in November, 2008. ${ }^{4}$ This power had been sought for more than 20 years by some professionals ${ }^{5}$ but was resisted by a coalition of 32 professional and patient organisations. ${ }^{6}$ CTOs require patients to accept clinical monitoring and allow rapid recall for assessment but do not authorise forcible treatment outside hospital. The existing provision for "leave of absence" under Section 17 of the Act, which confers similar powers over outpatient care, was retained. This allows patients to leave hospital for some hours or days, or even exceptionally weeks, while still subject to recall. Its purpose is to assess recovery before granting voluntary status. The existence of this alternative made randomisation on discharge legally and ethically possible.

Early opposition to CTOs focused on civil liberties ${ }^{8}$ or lack of improvement on the existing leave regime. ${ }^{9}$ More recent opposition has emphasised the absence of experimental evidence. ${ }^{10}$ There are several non-experimental studies of CTOs $^{10,11}$ but only two published randomised trials, ${ }^{12,13}$ both from the USA. Neither of their results showed a difference in the primary outcome measure of readmission rates.

Results from a secondary analysis of the North Carolina randomised tria ${ }^{13}$ showed significantly fewer readmissions

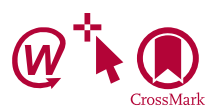

CrossMark

Published Online

March 26, 2013

http://dx.doi.org/10.1016

S0140-6736(13)60107-5

See Comment page 1603

Department of Psychiatry

(Prof T Burns DSc, J Rugkåsa PhD,

K Yeeles BSc), Department of

Primary Health Care Sciences

(M Vazquez-Montes PhD),

Centre for Statistics in

Medicine (M Voysey MBiostat),

University of Oxford, Oxford,

UK; Health Services Research

Unit, Akershus University

Hospital, Lørenskog, Norway

(J Rugkåsa); Oxford Health NHS

Foundation Trust, Oxford, UK

(A Molodynski MBChB); Faculty

of Law, University of Otago,

Otago, New Zealand

(J Dawson LLD); Faculty of

Medicine, University of

Southampton, Southampton

UK (J Sinclair DPhil); and Unit

for Social \& Community

Psychiatry, Queen Mary

University of London, London, UK (Prof S Priebe FRCPsych)

Correspondence to:

Prof Tom Burns, Department of Psychiatry, Warneford Hospital, University of Oxford,

Oxford OX3 7JX, UK

tom.burns@psych.ox.ac.uk 
and reduced hospital days in patients with sustained CTOs (more than 180 days) and regular clinical contact (more than three times per month) compared with voluntary controls..$^{13}$ CTOs in England and Wales are for up to 6 months in the first instance and renewable, and frequent clinical monitoring was anticipated in the 2007 amendment. ${ }^{14}$ Our trial tests whether CTOs reduce admissions when patients in both groups receive equivalent levels of clinical contact but different lengths of compulsory supervision. A cost-effectiveness analysis will be the subject of a separate paper.

\section{Methods}

\section{Patients and trial design}

OCTET is a single-outcome randomised trial that tests the hypothesis that patients with a diagnosis of psychosis discharged from hospital on CTOs will have a lower rate of readmission over 12 months than will those discharged on Section 17 leave. Eligible patients were those currently detained for inpatient treatment, aged 18-65 years (the standard age range for UK adult mental health services), diagnosed with psychosis, not subject to any other legal restrictions, able to give informed consent and considered suitable for supervised outpatient care by their clinical team. We report data obtained from medical notes and structured patient interviews at baseline and 12 months.

Ethical approval was granted by the Staffordshire National Health Service (NHS) Research Ethics Committee (reference 08/H1204/131).

Specialist mental health services in England are provided by area-based NHS Mental Health Trusts, each divided into catchment areas where community mental health teams (CMHTs) provided both community and inpatient care at the start of our trial. During recruitment, many Trusts separated inpatient and community services, and CTOs were increasingly initiated by specialist inpatient psychiatrists. ${ }^{15}$ Recruitment was from Nov 10 , 2008, to Feb 22, 2011. All 62 Trusts within a reasonable travelling distance were originally approached. Local research governance approval was pursued with 44 Trusts, and 32 of them, predominantly in the Midlands and southern England, recruited to the trial.

\section{Randomisation and masking}

Consenting participants were randomly assigned (ratio 1:1) by an independent statistician to be discharged from hospital either on CTO or Section 17 leave. Randomisation used random permuted blocks with lengths of two, four, and six, and stratified for sex (male or female),

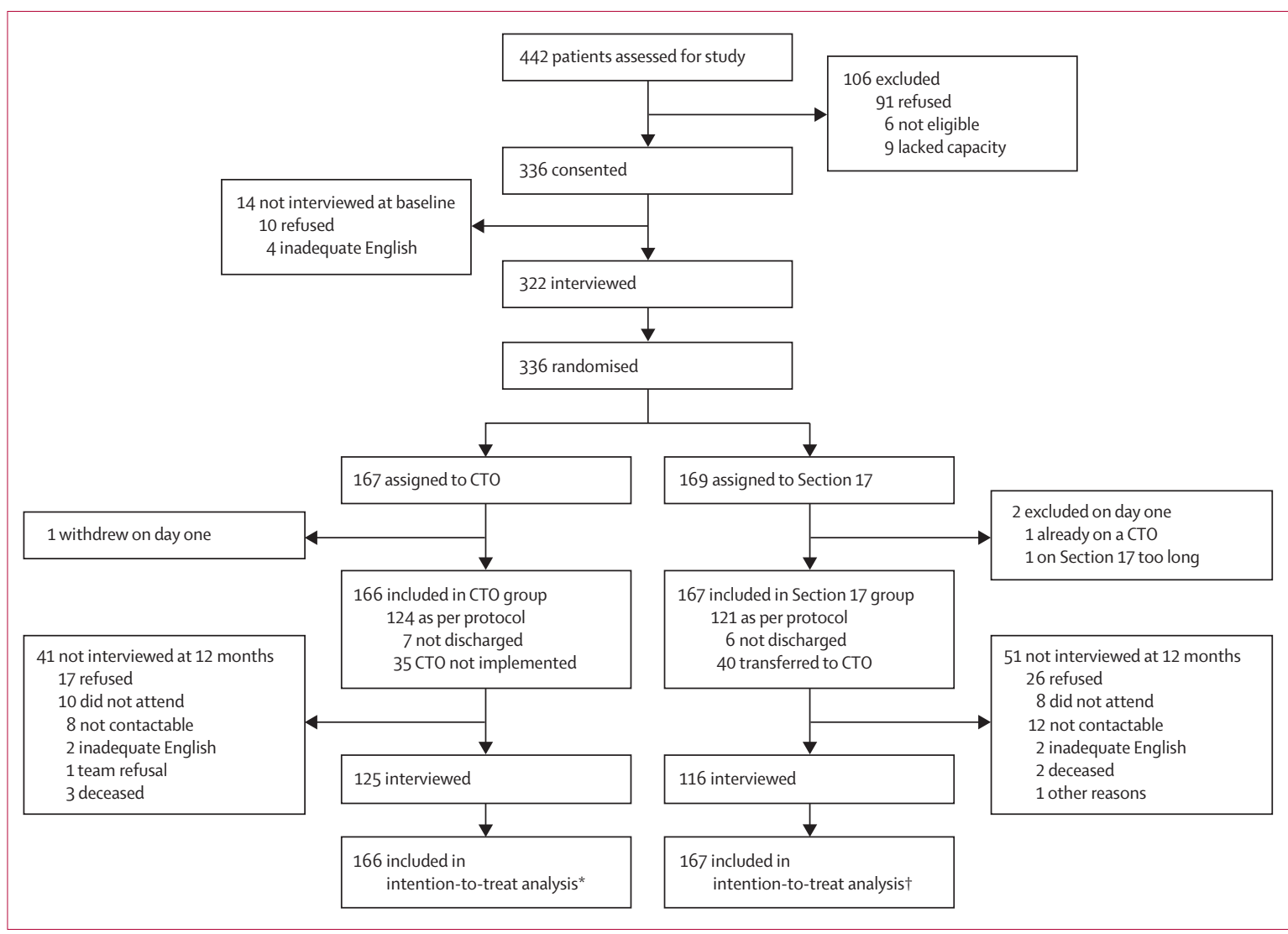

Figure 1: Trial profile

*Including three patients deceased during follow-up period. †Including two patients deceased during follow-up period. 
schizophrenic diagnosis (yes or no) and duration of illness $(<2$ years or $\geq 2$ years).

Assignments were enclosed in sequentially numbered, opaque, sealed envelopes and stored by a researcher independent to the trial team. The details of the sequence remained unknown to all members of the trial team until recruitment, data collection, and analyses were completed. Randomisation took place after consent was obtained and the baseline interview was done. The envelope was opened on the day of the interview by the independent researcher after recording the participant's trial identification number on the envelope. She then communicated the randomised allocation to the recruiting researcher by telephone.

As randomisation involved allocation to different legal statuses, it was both impossible and unlawful to mask research assistants, treating clinicians or patients.

\section{Procedures}

A CTO can be imposed when the responsible clinician (usually a consultant psychiatrist), and an approved mental health practitioner (usually a social worker) deem that a patient needs supervision after a period of involuntary hospital treatment and that, without it, is highly likely to relapse and be readmitted involuntarily. The approved mental health practitioner is required to consult with the patient and with family carers. The formal process is intentionally time consuming to ensure it is not used for clinical convenience. Several days elapse between the clinical decision and CTO activation.

It can be a stipulated condition of the CTO that the patient takes medication outside hospital, but the CTO does not authorise clinicians to administer it by force. It allows the responsible clinician to recall the patient for up to $72 \mathrm{~h}$ to review treatment without formally readmitting them. Other conditions can be imposed, such as place of residence and attendance at regular assessments. All conditions must be specified in the CTO documents. A CTO initially lasts up to 6 months, renewable for a further 6 months, and subsequently for 1-year terms. It can be discharged at any time by the responsible clinician or by a mental health tribunal if the patient's mental state or circumstances improve. During the CTO period, the hospital treatment order (Section 3) remains in place but is inactive; it is reactivated if the CTO is revoked after a recall to hospital.

Section 17 leave is a well established rehabilitation practice, used for brief periods to assess the stability of a patient's recovery after or during a period of involuntary hospital treatment. The treatment order (Section 3) remains active and the patient can be immediately readmitted without additional legal processes. Medication cannot be given by force in the community. Section 17 leave is extensively used but, as a continuation of Section 3, no routine data are collated. Its frequency and duration are therefore unknown but both are believed to be highly variable. Patients can be discharged directly from Section 3 without the need for either Section 17 leave or a CTO, and most are. Such patients would not be eligible for recruitment to this trial.

Our trial thus randomly assigned patients to two forms of mandatory outpatient care. A legal opinion on the trial concluded that, although there are undoubtedly differences between the CTO and leave regimes, an area of genuine legal equipoise exists; it is unclear whether either regime is more restrictive than the other and it is possible for a patient simultaneously to meet the criteria for both. ${ }^{\text {? }}$

\section{Statistical analysis}

The primary, binary outcome measure was whether or not the patient was admitted to hospital during the 12-month follow-up period. Secondary hypotheses were that CTOs would prolong time to first readmission, reduce the number of admissions and overall time in hospital, and improve clinical and social functioning. The secondary hospitalisation outcome measures were time to first readmission, number of days in hospital, and number of readmissions during follow-up. We assessed clinical outcome using the Brief Psychiatric Rating Scale

\begin{tabular}{|c|c|c|c|}
\hline & $\begin{array}{l}\text { Missing data } \\
(n=336)\end{array}$ & $\begin{array}{l}\text { СТо } \\
(n=167)\end{array}$ & $\begin{array}{l}\text { Section } 17 \\
(n=169)\end{array}$ \\
\hline \multicolumn{4}{|l|}{ Demographics } \\
\hline Age (years) & $0(0 \%)$ & $39 \cdot 8(11 \cdot 2)$ & $39 \cdot 5(11 \cdot 7)$ \\
\hline Male sex & $0(0 \%)$ & $111(66 \%)$ & $114(67 \%)$ \\
\hline Years of education & $4(1 \%)$ & $11 \cdot 8(1.7)$ & $12 \cdot 0(2 \cdot 1)$ \\
\hline Ethnic origin & $0(0 \%)$ & & \\
\hline White &.. & $102(61 \%)$ & $104(62 \%)$ \\
\hline Black &.. & $38(23 \%)$ & $40(24 \%)$ \\
\hline Asian &. & $15(9 \%)$ & $14(8 \%)$ \\
\hline Mixed and other & .. & $12(7 \%)$ & $11(7 \%)$ \\
\hline Born in UK & $1(<1 \%)$ & $135(81 \%)$ & $124(74 \%)$ \\
\hline \multicolumn{4}{|l|}{ Living situation } \\
\hline Married or cohabiting & $2(<1 \%)$ & $11(7 \%)$ & $18(11 \%)$ \\
\hline Identified carer & $30(9 \%)$ & $62(41 \%)$ & $50(32 \%)$ \\
\hline Independent accommodation & $2(<1 \%)$ & $118(71 \%)$ & $123(73 \%)$ \\
\hline Living alone or homeless & $18(5 \%)$ & $123(79 \%)$ & $116(72 \%)$ \\
\hline \multicolumn{4}{|l|}{ Clinical status } \\
\hline Schizophrenia & $0(0 \%)$ & $141(84 \%)$ & $145(86 \%)$ \\
\hline BPRS & $22(7 \%)$ & $38(30-48)$ & $38(32-49)$ \\
\hline GAF & $25(7 \%)$ & $38 \cdot 3(9 \cdot 4)$ & $39 \cdot 3(10 \cdot 2)$ \\
\hline \multicolumn{4}{|l|}{ Clinical history } \\
\hline Duration of illness (years) & $9(3 \%)$ & $12(6-20)$ & $12 \cdot 5(5-21)$ \\
\hline Less than 2 years illness duration & $0(0 \%)$ & $7(4 \%)$ & $7(4 \%)$ \\
\hline Number of past psychiatric hospital admissions & $22(7 \%)$ & $6(3-8)$ & $5(3-9)$ \\
\hline Duration of past psychiatric hospital admissions (months) & $58(17 \%)$ & $14(6-28)$ & $15(7-30)$ \\
\hline Number of past involuntary hospital admissions & $33(10 \%)$ & $4(2-7)$ & $3(2-8)$ \\
\hline Criminal conviction & $31(9 \%)$ & $65(43 \%)$ & $68(44 \%)$ \\
\hline Previous imprisonment & $24(7 \%)$ & $41(27 \%)$ & $45(28 \%)$ \\
\hline $\begin{array}{l}\text { Data are number (\%), mean (SD), or median (IQR). CTO=comm } \\
\text { scale. GAF=global assessment of functioning. }\end{array}$ & y treatment orde & s. BPRS=brief ps & chiatric rating \\
\hline
\end{tabular}




\begin{tabular}{|c|c|c|c|c|c|c|c|}
\hline & \multicolumn{3}{|c|}{ СТО $(n=166)$} & \multicolumn{3}{|c|}{ Section $17(n=167)$} & \multirow[t]{2}{*}{ Treatment effect } \\
\hline & $\mathrm{N}$ & $\begin{array}{l}\text { Number (\%) or } \\
\text { mean (SD) }\end{array}$ & Median (IQR) & $\mathrm{N}$ & $\begin{array}{l}\text { Number (\%) or } \\
\text { mean (SD) }\end{array}$ & Median (IQR) & \\
\hline \multicolumn{8}{|l|}{ Primary outcome } \\
\hline Psychiatric hospital readmission & 166 & $59(36 \%)$ & .. & 167 & $60(36 \%)$ & .. & $1.00(0.75 \text { to } 1.33)^{*}$ \\
\hline \multicolumn{8}{|l|}{ Secondary outcomes } \\
\hline $\begin{array}{l}\text { Total duration of all psychiatric } \\
\text { hospitalisations (nights) }\end{array}$ & 166 & $82 \cdot 2(102 \cdot 0)$ & $41 \cdot 5(8-109)$ & 167 & $90 \cdot 9(104 \cdot 5)$ & $48(10-133)$ & $0.90(0.65$ to 1.26$) \dagger$ \\
\hline Number of readmissions & 59 & $1.2(0 \cdot 6)$ & $1(1-1)$ & 60 & $1.4(0.8)$ & $1(1-2)$ & $0.82(0.58$ to 1.16$) \dagger$ \\
\hline Number with multiple readmissions & 166 & $10(6 \cdot 0 \%)$ & .. & 167 & $18(10 \cdot 8 \%)$ & .. & .. \\
\hline Days in community until first readmission & 166 & $246(122 \cdot 4)$ & $295(140-357)$ & 167 & $241(126 \cdot 5)$ & $292(126-354)$ & $\mathrm{p}=0.755 \ddagger$ \\
\hline \multicolumn{8}{|l|}{ Clinical outcomes } \\
\hline BPRS (at 12 months) & 122 & $38 \cdot 2(11 \cdot 5)$ & $35(29-44)$ & 112 & $38 \cdot 3(12 \cdot 4)$ & $34(30-43 \cdot 5)$ & $-1.09(-3.25$ to 1.07$) \sqrt{5}$ \\
\hline GAF (at 12 months) & 123 & $39.0(12 \cdot 0)$ & $36(31-45)$ & 114 & $39 \cdot 7(13 \cdot 1)$ & $35 \cdot 5(30-45)$ & $-0.86(-2.93$ to 1.20$)$ S \\
\hline
\end{tabular}

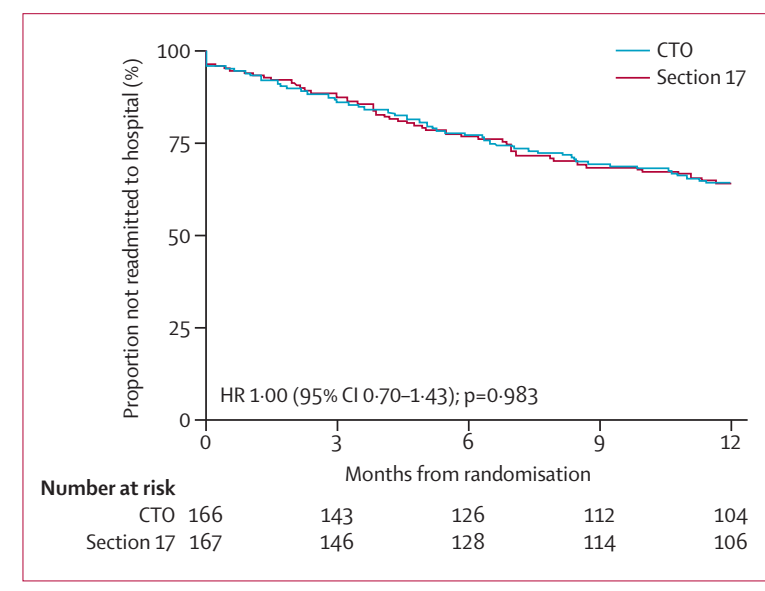

Figure 2: Time to readmission $\mathrm{CTO}=$ community treatment orders.

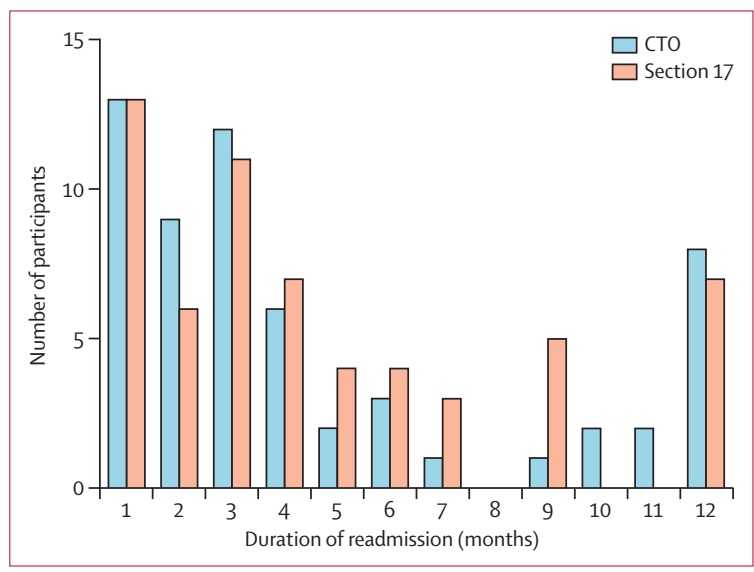

Figure 3: Duration of admissions in months $\mathrm{CTO}=$ community treatment orders.
(BPRS), a well established scale rating 24 symptoms from 1 (absent) to 7 (extremely severe). ${ }^{16}$ We assessed social outcome using the Global Assessment of Functioning (GAF), which gives a single score between 1 (for a severe life threatening state) to 100 (for superior functioning). ${ }^{17}$ The scale has clearly anchored deciles and established reliability. We used both scales at baseline and 12 months, administered by independent, trained researchers .

A sample size of 288 patients was needed to detect a difference of $16 \%$ in the proportion readmitted to a psychiatric hospital between the two groups $(48 \%$ in section 17 vs $32 \%$ on CTO) as in the North Carolina trial, ${ }^{13}$ assuming $\alpha$ of $5 \%$ and power of $80 \%$. We predicted attrition to be negligible as primary outcome data were available from medical records.

Two interim analyses, prepared by an independent statistician, and masked to the investigators, were submitted to an independent data monitoring committee who agreed unanimously that it was safe to continue the trial.

The trial team wrote and signed off a detailed statistical analytical plan before any data were analysed. All analyses were done according to the analysis plan.

We analysed the primary outcome using a log-binomial regression model adjusted for stratification factors (sex, schizophrenic status, and duration of illness). The result is presented as an adjusted relative risk with 95\% CI. We analysed count outcomes such as number of nights in psychiatric hospital admission and number of readmissions using adjusted negative-binomial or zero-inflated Poisson regression models dependent on data distribution. We present the results as adjusted incident-density ratios (IDR) with 95\% CIs and interpret them in a similar way as relative risks.

We analysed time-to-event outcomes such as time from randomisation to readmission using proportional hazards regression models and present the results as adjusted hazard ratios (HRs) with 95\% CIs along with 


\begin{tabular}{|c|c|c|c|c|c|}
\hline & \multicolumn{2}{|l|}{ CTO $(n=166)$} & \multicolumn{2}{|c|}{ Section $17(n=167)$} & \multirow[t]{2}{*}{ Treatment effect } \\
\hline & Mean (SD) & Median (IQR) & Mean (SD) & Median (IQR) & \\
\hline Days under any legal compulsion during follow-up & $241 \cdot 4(121 \cdot 1)$ & $255(148-365)$ & $134 \cdot 6(114 \cdot 1)$ & $102(31-212)$ & $p<0.0001^{*}$ \\
\hline Days in community under randomised legal compulsion & $170 \cdot 1(134 \cdot 4)$ & $183(0-299)$ & $45 \cdot 5(80 \cdot 7)$ & $8(0-37)$ & $p<0.0001^{*}$ \\
\hline Number of contacts with service (per month) & $3 \cdot 0(3 \cdot 1)$ & $2 \cdot 1(0 \cdot 8-4 \cdot 4)$ & $3 \cdot 9(5 \cdot 9)$ & $2 \cdot 2(0.8-4 \cdot 7)$ & $0.77(0.47-1.26) \dagger$ \\
\hline \multicolumn{6}{|c|}{ Data are mean (SD) or median (IQR). *Wilcoxon rank-sum p value. tIncident density ratio (95\% Cl). } \\
\hline
\end{tabular}

Kaplan-Meier plots. We compared other outcomes with distributions that violated assumptions for all the above methods using Wilcoxon rank-sum tests.

We used mean imputation to calculate BPRS total score for cases with at least ten items completed. For BPRS and GAF, we estimated treatment effect and change over time using a hierarchical model with random patient intercept adjusting for stratification factors. We did all analyses according to the intention-totreat principle. We performed sensitivity analyses, which were unadjusted for stratification factors. We used Stata version 12.1 for all analyses. This trial is registered, number ISRCTN73110773.

\section{Role of the funding source}

The views expressed in this presentation are those of the authors and not necessarily those of the NHS, the NIHR, or the Department of Health. The sponsor of the trial had no role in trial design, data collection, data analysis, interpretation, or writing of the report. The corresponding author had full access to all the data in the trial and had final responsibility for the decision to submit for publication.

\section{Results}

Of 442 patients assessed by the study team, 336 patients were randomly assigned to receive either CTO (167 patients) or Section 17 (169 patients; figure 1). Table 1 shows baseline characteristics. One patient withdrew and two were identified as ineligible directly after randomisation (figure 1). Data for the primary outcome measure were available from medical records for all 333 patients. 241 (72\%) patients were interviewed at 12 months and baseline characteristics did not differ between those who were interviewed and those who were not (appendix p 1). Five people died during follow-up: two deaths by suicide, and one by accidental death from a drug overdose were recorded in the CTO group, and one death by suicide and one death from natural causes were recorded in the Section 17 leave group.

Primary, secondary, or clinical outcomes did not differ between groups (table 2). At 12 months, neither the number of patients readmitted, nor the time to readmission from randomisation, nor the number of days in hospital differed (table 2; figure 2). The pattern of duration of individual admissions seemed similar for both groups (figure 3). Neither symptoms as measured by BPRS nor social functioning as measured by GAF differed between groups at 12 months (table 2). A sensitivity analysis excluding those who did not receive CTO and those who went directly from Section 17 to CTO gave similar results.

The duration of time in the community after hospital discharge under initial randomised compulsory outpatient supervision (ie, randomised compulsion) was substantially longer in the CTO group (table 3); as was the time to first voluntary status (appendix p 2). Time from randomisation to discharge from hospital was similar between groups (median 8 days in the CTO group vs 16 days in the Section 17 group, HR 1.08 (95\% CI 0.86-1·34; appendix pp 3-4). Overall, the total number of days under compulsion during follow-up was significantly greater in the CTO group (median 255 days) than in the Section 17 group (102 days; $\mathrm{p}<0 \cdot 0001$ ). This total number of days included compulsion under the randomised regime (either CTO or Section 17), any time on CTOs for crossover patients in the Section 17 group, and any time on a subsequent CTO or Section 17 for patients who came under these regimes more than once during follow-up. Self-reported monthly rate of clinical contacts during the time outside hospital did not differ between the two groups (table 3).

\section{Discussion}

Our results do not support our hypothesis. Despite a more than three-fold increase in time under initial supervised community care, the rate of readmission to hospital was not decreased by CTOs. Neither was the time to readmission decreased nor was there any significant difference in the number or duration of hospital admissions. We also recorded no differences in clinical or social outcomes.

We chose the rate of readmission as our primary outcome, and powered our trial on it, because CTOs have been legislated explicitly for this outcome, and it is also the primary outcome in most major studies, ${ }^{10,11,18}$ including the only two published randomised trials (panel). ${ }^{12,13}$ The total number of days in psychiatric hospital was very similar for both groups in our trial, as was the time from randomisation to initial discharge. Several patients remained in hospital for most of the trial period, including 13 who were never discharged, because of deteriorations of their clinical state, and who had no opportunity to be supervised in the community. Patterns 


\section{Panel: Research in context}

\section{Systematic review}

Two detailed reviews ${ }^{10,11}$ have been published of research into community treatment orders (CTOs) covering more than 70, mainly observational, studies and local clinical assessments. Only two randomised trials have been published ${ }^{12,13}$ and neither found any clear advantage to CTOs in terms of reducing hospital admission in patients with a diagnosis of psychosis. Both randomised trials are more than a decade old and were done in the USA. Since then, CTOs have been introduced in several European countries including England and Wales in 2008

\section{Interpretation}

Results from our trial showed no difference in the proportion of patients readmitted to hospital between study groups, nor in the time to readmission over a 1-year follow-up. The overall duration of hospital care did not decrease nor did clinical or social functioning improve despite an average of 6 months additional compulsion. These findings confirm previous evidence that CTOs do not confer benefits on patients with a diagnosis of psychosis, and their current high usage should be urgently reviewed.

of care will be carefully examined in subsequent health economic analyses.

The number of protocol violations is noteworthy (42 violations in the CTO group and 46 in the Section 17 group). Three factors contributed to this result. First, the legal position underpinning the lawfulness of the trial required each subsequent clinical decision to be entirely unconstrained by the randomisation. ${ }^{7}$ Consequently, clinicians could not be encouraged to persist with the initial randomised assignment. Second, recruitment of patients was at the point that the clinical team decided to initiate supervised outpatient care. Finalisation of a CTO requires detailed consultation both within the team and with the patient and their family, which can take from a few days to several weeks. Third, adherence to randomisation was compromised by reorganisation in almost all of the collaborating mental health services, particularly by the separation of inpatient and outpatient consultant responsibility. As a result, many patients passed to being treated by psychiatrists unfamiliar with the trial and who might have had different opinions about management. A sensitivity analysis removing these protocol violations did not alter our findings.

A limitation of testing the impact of a new intervention at its outset is a possible slow acquisition of confidence and skills in its use, thereby reducing trial generalisability. The median duration of the initial CTOs in our trial was 6 months, indicating that about half were being renewed. Combined with clinical contacts averaging over two per month, this result suggests that CTOs are being implemented in line with the legislators' expectations.
Figures for the average duration of CTOs in England and Wales are not available. Centrally obtained data are difficult to interpret but seem broadly in line with the pattern observed in our trial. Of the 14295 CTOs issued from 2008-12, 3922 have been discharged, 3409 revoked, and 4764 reported to be in place at the end of 2012. For each of the last 3 years, about 4000 new CTOs have been issued, with between 2000 and 3000 revoked or discharged, the remainder presumably being renewed or allowed to lapse as patients are judged to need them no longer. ${ }^{19}$

Only patients deemed suitable for a CTO were eligible for our trial, and many patients were discharged from Section 3 with a period of Section 17 without being included. The legal requirements for the trial restricted eligible patients to those deemed "suitable for discharge either to Section 17 leave or CTO”. Collaborating clinicians accepted that in the absence of convincing evidence of efficacy, any patient they deemed suitable for CTO was also eligible for leave. Those who believed otherwise (or were committed to a routine use of protracted Section 17 leave as an alternative) did not recruit to the trial. It was not possible to obtain information about those patients on whom collaborating clinicians imposed CTOs without entering them into the trial. This certainly did happen on several occasions, especially when patients' families had expressed strong preferences. The exclusion of obvious candidates for CTO could potentially bias against proving an effect of CTOs, but this bias is impossible to estimate.

This is the third, and largest, randomised trial of CTOs, and, similar to its predecessors, did not find any evidence that CTOs achieve their intended purpose of reducing readmission in so-called revolving door patients with a diagnosis of psychosis. The evidence is now strong that the use of CTOs does not confer early patient benefits despite substantial curtailment of individual freedoms. In view of the now consistent experimental evidence against any clear benefit, we believe any proposal to either introduce CTOs to new jurisdictions or extend their use would require a commitment to test their effects at least as rigorously as we have done. Major ethical and legal barriers have to be overcome to do such research., ${ }^{7,21}$ International experience indicates that clinicians rapidly form strong opinions for or against CTOs and clinical equipoise is soon lost. ${ }^{22}$ However, as previously argued, the introduction of such restrictions on patient liberties would be unethical unless accompanied by a rigorous assessment of their potential costs and benefits. ${ }^{23}$

\section{Contributors}

TB designed the trial with input from SP and JS. All authors were involved in the conduct of the trial, interpretation of results, and revision and correction of the report, which was drafted by TB, JR, and JD. The analyses were led by MV-M. All authors read and approved the final version of the report.

\section{The OCTET Group}

Caroline Bennet, Kiki Burns, Tom Burns, Krysia Canvin, John Dawson, Alastair Gray, Lindsey Johnson, Naomi Lewis, Sarah Masson, Andrew Molodynski, Lucy Murray, Helen Nightingale, Riti Patel, 
Stefan Priebe, Stephen Puntis, Jorun Rugkåsa, Lisa Russell,

Aonghus Ryan, Judit Simon, Julia Sinclair, Tanya Smyth, Anna Sulman, Lucinda Turnpenny, Maria Vazquez Montes, Francis Vergunst, Claire Visser, Merryn Voysey, and Ksenija Yeeles.

\section{Conflicts of interest}

We declare that we have no conflicts of interest.

\section{Acknowledgments}

The trial is funded by a Programme Grant for Applied Research from the National Institute of Health Research (RP-PG-0606-1006). It is adopted into the UKCRN and MHRN portfolios, and is registered with ISRCTN [ISRCTN73110773]. The views expressed in this publication are those of the authors and not necessarily those of the NHS, the NIHR, or the Department of Health.

\section{References}

1 Torrey EF, Entsminger K, Geller J, Stanley J, Jaffe DJ. The shortage of public hospital beds for mentally ill persons. Virginia: Treatment Advocacy Center, 2008.

2 Appelbaum PS. Thinking carefully about outpatient commitment. Psychiatr Serv 2001; 52: 347-50.

3 Priebe S, Badesconyi A, Fioritti A, et al. Reinstitutionalisation in mental health care: comparison of data on service provision from six European countries. BMJ 2005; 330: 123-26.

4 Department of Health. Mental Health Act 2007: patients on aftercare under supervision (ACUS): transitional arrangements. London: Department of Health, 2008.

5 Royal College of Psychiatrists. Community Supervision Orders. Council Report CR18. London: Royal College of Psychiatrists, 1993.

6 The Mental Health Alliance. Towards a better Mental Health Act. London: The Mental Health Alliance, 2005.

7 Dawson J, Burns T, Rugkåsa J. Lawfulness of a randomised trial of the new community treatment order regime for England and Wales. Med Law Rev 2011; 19: 1-26.

8 Munetz MR, Galon PA, Frese FJ 3rd. The ethics of mandatory community treatment. J Am Acad Psychiatry Law 2003; 31: 173-83.

9 Gledhill K. Community treatment orders. J Ment Health 2007; L: $149-69$.

10 Churchill R, Owen G, Singh S, Hotopf M. International experiences of using Community Treatment Orders. London: Institute of Psychiatry, 2007.
11 Dawson J. Community treatment orders: international comparisons. Dunedin: Otago University, 2005.

12 Steadman HJ, Gounis K, Dennis D, et al. Assessing the New York City involuntary outpatient commitment pilot program. Psychiatr Serv 2001; 52: 330-36.

13 Swartz MS, Swanson JW, Wagner HR, Burns BJ, Hiday VA, Borum R. Can involuntary outpatient commitment reduce hospital recidivism?: findings from a randomized trial with severely mentally ill individuals. Am J Psychiatry 1999; 156: 1968-75.

14 Department of Health. Code of Practice: Mental Health Act 1983. London: Stationery Office, 2008.

15 Burns T. The dog that failed to bark. The Psychiatrist 2010; 34: 361-63.

16 Lukoff D, Nuechterlein K, Ventura J. Manual for expanded brief psychiatric rating scale (BPRS). Schizophr Bull 1986; 12: 594-602.

17 Goldman HH, Skodol AE, Lave TR. Revising axis V for DSM-IV: a review of measures of social functioning. Am J Psychiatry 1992; 149: $1148-56$

18 Kisely SR, Campbell LA, Preston NJ. Compulsory community and involuntary outpatient treatment for people with severe mental disorders. Cochrane Database Syst Rev 2005; 3: CD004408.

19 Health and Social Care Information Centre, Community and Mental Health Team. Inpatients formally detained in hospitals under the Mental Health Act 1983, and patients subject to supervised community treatment, Annual figures, England 2011/12. Leeds: Health and Social Care Information Centre; 2012.

20 Dawson J. Randomised controlled trials of mental health legislation. Med Law Rev 2002; 10: 308-21.

21 Swartz MS, Burns BJ, George LK, et al. The ethical challenges of a randomized controlled trial of involuntary outpatient commitment. J Ment Health Adm 1997; 24: 35-43.

22 Romans S, Dawson J, Mullen R, Gibbs A. How mental health clinicians view community treatment orders: a national New Zealand survey. Aust N Z J Psychiatry 2004; 38: 836-41.

23 Burns T, Dawson J. Community treatment orders: how ethical without experimental evidence? Psychol Med 2009; 39: 1583-86. 